\title{
WHITE CATARACT - CHALLENGES AND SOLUTIONS
}

\author{
Dessislava Stateva
}

\author{
Eye Clinic, Medical University - Pleven
}

\begin{abstract}
INTRODUCTION: Cataract surgery is the most common ophthalmological surgical intervention. It may have different outcomes depending on the cataract type and the accompanying eye or systemic diseases.

MATERIAL AND METHODS: A total of 41 white cataract cases were reviewed. Preoperative examination included visual acuity, detailed slitlamp evaluation, applanation tonometry, keratometry, and axial length measurements.
\end{abstract}

RESULTS: Among our 41 cases there was a slight prevalence of males and urban citizens. The greatest challenge was the capsulorhexis. For its successful completion we used heavy viscous substance and capsulotome.

CONCLUSION: In spite of the precise preoperative examination white cataract could "surprise" the surgeon during the course of the operation.

Keywords: white cataract, phacoemulsification, continuous curvilinear capsulorhexis

\section{INTRODUCTION}

Cataract is a major cause of treatable blindness worldwide (1). The only treatment is surgical. It is achieved via phacoemulsification and is fast, efficient and painless. However, every surgery has potential risks and every case is unique. So every cataract case has to be evaluated prior to surgery and the possible risks and complications should be estimated.

Address for correspondence:

Dessislava Stateva, $M D, P h D$

Eye Clinic, Medical Univerisity - Pleven

91 General Vladimir Vazov Str.

5800 Pleven, Bulgaria

e-mail:dessy_veleva@yahoo.com

Received: November 11, 2014

Accepted: December 19, 2014

\section{MATERIAL AND METHODS}

Medical records of cataract patients admitted to the Eye Clinic of University Hospital - Pleven for 2 year period were reviewed. A total of 41 white cataract cases were found. Preoperative examination included visual acuity, detailed slitlamp evaluation, applanation tonometry, keratometry, and axial length measurements. All patients were operated by the same surgeon. Retrobulbar anesthesia was used. Phacoemulsification was the surgical method in all cases. The total phaco time was recorded. Foldable IOLs were implanted.

\section{RESULTS}

Out of the 41 patients with white cataract, $27(66 \%)$ were urban citizens and $14(34 \%)$ were rural citizens. Twenty three patients were male and 18 were female. The mean age was 68.18 years (the youngest patient was 51 and the oldest was 91 years old).

Accompanying eye diseases were: glaucoma in 4 cases and corneal dystrophy in one case. Pseudo- 
exfoliation syndrome was found in 2 cases. Diabetes was found in 3 cases.

Preoperative visual acuity of all patients was LP ("light perception"). On the first day after surgery 24(59\%) patients had normal ocular status. The other $17(41 \%)$ patients had corneal edema and/or Descemet membrane folds. Visual acuity one week after surgery was better than 0.1 in $30(73 \%)$ cases.

Intraoperative complications were rare - in one case the capsulorhexis was finished with scissors because of a fibrous capsule and in another case there was rupture of the posterior capsule and the IOL was implanted in the sulcus.

\section{DISCUSSION}

Terms like mature, hypermature, advanced or intumescent cataract are usually used to describe white cataract (2). The surgeon may come upon various nuclear hardness and has to be prepared for any surgery outcomes. That is why retrobulbar anesthesia is preferred over topical anesthesia.

The greatest challenge of white cataract is to complete the continuous curvilinear capsulorhexis (Figure 1).

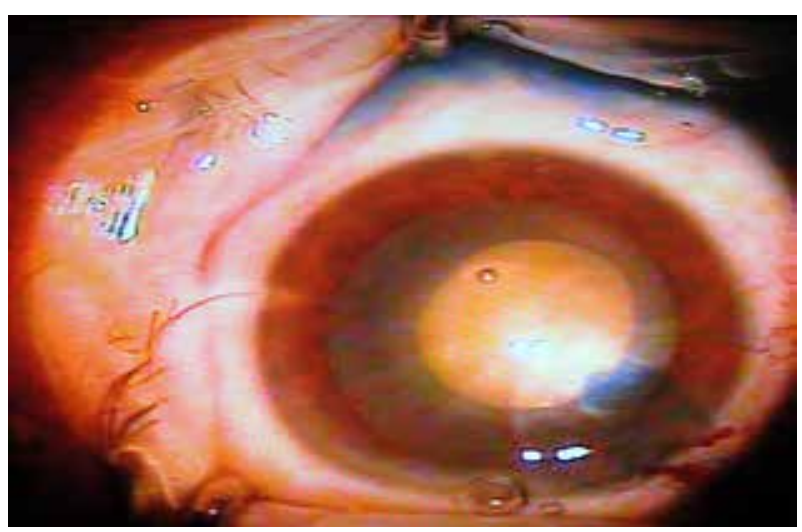

Fig. 1. Continuous curvilinear capsulorhexis

For that purpose a dye is required - to color the anterior capsule (Figure 2) for better visualization (because there is no fundus reflex) $(3,4)$. At the start of the capsulorhexis "Argentinian flag" sign may occur - a state where the anterior capsule splits right after the puncture and looks like the blue-white Argentinian flag. This is caused by increased intracapsular pressure in the capsule which may result in sudden radial extension of the anterior capsulotomy to-

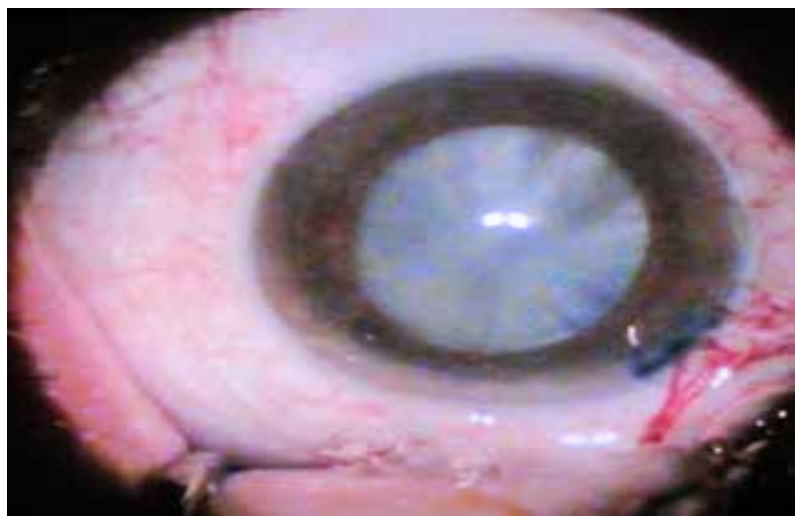

Fig. 2. Blue colored anterior capsule

wards the periphery (5). Another possible scenario is the front chamber to be filled with "milky" content. These complications could be avoided with the use of proper viscous substance, very small puncture in the anterior capsule and extraction of some of its liquid content. Thus the tension will be reduced and the chances to perform a continuous capsulorhexis will increase. These considerations are the reason why the surgeon often has to deal with hard nucleus under the conditions of small capsulorhexis.

In patients with a pseudoexfoliation syndrome another obstacle for the surgeon is small pupil. In these cases iris retractors should be used. We had one such case (Figure 3 and 4).

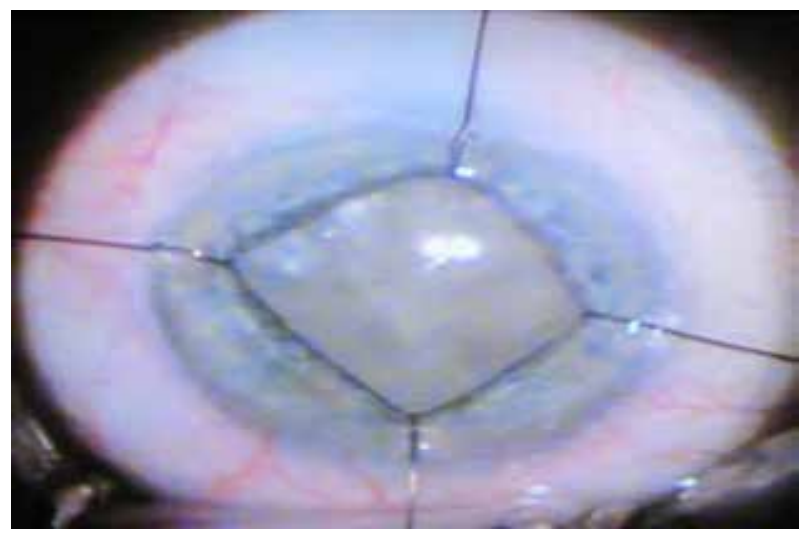

Fig. 3. Iris retractors

The most common complication of phacoemulsification is rupture of the posterior capsule (6). It occurs more often among white cataract patients and may be accompanied by loss of vitreous and "sinking" of the nucleus. This may force the surgeon to switch to extracapsular cataract extraction (ECCE) 


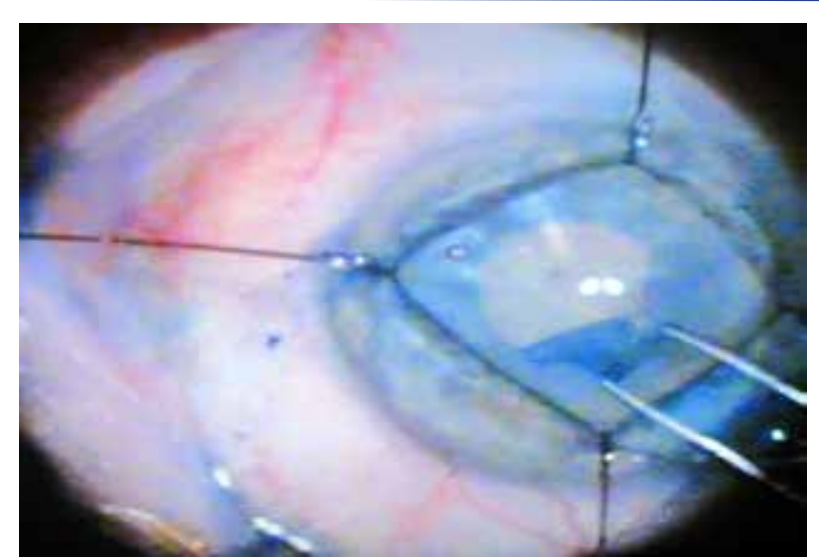

Fig. 4. Iris retractors, blue colored anterior capsule and beginning of capsulorhexis

(7). In one case out of all 41 we had to complete the operation as ECCE.

If the surgeon had to extract a hard nucleus there could be an increased intraocular pressure and damage to the cornea. Because of this such patients require more frequent follow-up.

The prognosis for the visual acuity in white cataract cases is unclear regardless of the preoperative B-scan (8). This requires thorough postoperative fundus examination.

\section{CONCLUSION}

White cataract presents a challenge even for the most experienced phaco-surgeon. However, a good preoperative case evaluation may minimize the intraoperative risks and result in a "happy surgery outcome" both for patient and surgeon.

\section{REFERENCES}

1. Xu L, Cui T, Zhang S, Sun B, Zheng Y, Hu A, Li J, $\mathrm{Ma} \mathrm{K}$, Jonas JB. Prevalence and risk factors of lens opacities in urban and rural Chinese in Beijing. Ophthalmology, 2006;113(5):747-755.

2. Pineda R, Espaillat A, Perez VL, Rowe SG. The complicated cataract. The MEEI phacoemulsification practice handbook. Boston: Slack. 2001. 163 p.

3. Jacobs DS, Cox TA, Wagoner MD, Ariyasu RG, Karp CL. Capsule staining as an adjunct to cataract surgery: a report from the American Academy of Ophthalmology. Ophthalmology, 2006;113(4):707-713.

4. Dada VK, Sharma N, Sudan R, Sethi H, Dada T, Pangtey MS. Anterior capsule staining for capsulorhexis in cases of white cataract: compar- ative clinical study. J Cataract Refract Surg, 2004;30(2):326-333.

5. Vasavada A, Singh R, Desai J. Phacoemulsification of white mature cataracts. J Cataract Refract Surg, 1998;24(2):270-277.

6. Greenberg PB, Tseng VL, Wu WC, Liu J, Jiang L, Chen CK, Scott IU, Friedmann PD. Prevalence and predictors of ocular complications associated with cataract surgery in United States veterans. Ophthalmology, 2011;118(3):507-514.

7. Chakrabarti A, Singh S. Phacoemulsification in eyes with white cataract. J Cataract Refract Surg, 2000;26(7):1041-1047.

8. Rosenfeld SI, Johns KJ eds. Basic and clinical science course: Section 11 Lens and Cataract. San Francisco: American Academy of Ophthalmology. 2004. $176 \mathrm{p}$. 